Research Article

\title{
Receptivity of the Boundary Layer over a Blunt Wedge with Distributed Roughness at Mach 6
}

\author{
Zhenqing Wang $\mathbb{D}^{1},{ }^{1}$ Mingfang Shi $\mathbb{D}^{1},{ }^{1}$ Xiaojun Tang $\mathbb{D}^{2},{ }^{2}$ Lidan $X u^{1}$ and Xiaokun Sun ${ }^{1}$ \\ ${ }^{1}$ College of Aerospace and Civil Engineering, Harbin Engineering University, Harbin 150001, China \\ ${ }^{2}$ China Academy of Space Technology, Beijing 100094, China
}

Correspondence should be addressed to Xiaojun Tang; xiaojuntang87@sina.com

Received 8 December 2017; Accepted 28 March 2018; Published 29 April 2018

Academic Editor: William W. Liou

Copyright ( 2018 Zhenqing Wang et al. This is an open access article distributed under the Creative Commons Attribution License, which permits unrestricted use, distribution, and reproduction in any medium, provided the original work is properly cited.

\begin{abstract}
A hypersonic flow field over a blunt wedge with or without roughness is simulated by a direct numerical simulation method. The effect of isolated and distributed roughnesses on the steady and unsteady hypersonic flow field and boundary layer is analyzed. The shape of roughness is controlled by cubic polynomial. The evolution of disturbance waves caused by slow acoustic wave in the boundary layer is investigated by fast Fourier spectrum analysis. The results show that there is a great influence of roughness on the evolution of disturbance waves in the hypersonic boundary layer. The disturbance waves are promoted in the upstream-half region of roughness while suppressed in the downstream-half region of roughness. There is always a mode competition among different modes both in the temporal domain and in the frequency domain in the boundary layer, and mode competition is affected by roughness. The location of the dominant mode which is changed to a second-order harmonic mode from the fundamental mode moves upstream. The vortices caused by roughness also impact the evolution of disturbance waves in the boundary layer. The fundamental mode is suppressed in the vortex region while other harmonic modes are promoted.
\end{abstract}

\section{Introduction}

Boundary-layer laminar-turbulent transition effectively affects the aerodynamic lift and drag of hypersonic vehicles and has a great effect on the design of hypersonic vehicles [1-3]. However, the process of transition is very complex and affected by many factors. In general, there are four phases of transition under freestream with small disturbances: (1) receptivity, (2) linear instability, (3) nonlinear stability and saturation, and (4) secondary instability and breakdown to turbulence. The receptivity is crucial in the process of transition because it defines the initial state of the disturbances in the boundary layer. Receptivity of the boundary layer is the response process of the boundary layer to external disturbances that include freestream disturbances, wall temperature, and roughness.

Investigating the effect of freestream disturbances is significant to understand the transition mechanism. The type and parameters of freestream disturbances both affect the process of transition, and it has been investigated by many scholars [4-11]. As the amplitude of freestream disturbances increases to finite amplitude, the second and third phases of transition may be short or even disappear. The transition mechanism is essentially changed by the change of amplitude of freestream disturbances. Ma and Zhong $[4,5]$ investigate the receptivity over a plate flat under the freestream with small disturbances by DNS and linear stability theory (LST). Balakumar and Kegerise [6] analyze the response of the boundary layer over straight and flared cones to small disturbances. Small disturbances and finite amplitude disturbances $\left(10^{-4}-10^{-1}\right)$ in the boundary layer over the blunt wedge are both investigated to reveal the leading edge receptivity by Cerminara and Sandham [7], and the Fourier analysis method is used to analyze the evolution of disturbances in the boundary layer. D. Park and S. O. Park [8] and Olaf et al. [9] also investigate the evolution process under the wall roughness in nonlinear instability by Fourier spectrum analysis. As for the type of freestream disturbances, they can be decomposed into entropy, vortical, and acoustic waves which include slow acoustic waves and fast acoustic waves. Qin and $\mathrm{Wu}$ [10], Zhang et al. [11], and Ma and Zhong [5] research the receptivity characteristic of a supersonic/hypersonic 
boundary layer under small disturbances with different types of freestream disturbances and found that there is a great difference between the four kinds of disturbances on the transition of the boundary layer.

In view of the fact that the change of flow state in the hypersonic boundary layer directly affects the propulsion efficiency and maneuverability of the hypersonic vehicle, and results in aerodynamic drag and significantly increased wall heating, laminar flow control (LFC) technologies are addressed. Laminar flow control technologies can be divided into three categories [12]: (1) active techniques: such as reverse jet [13], local heating, or cooling; (2) passive techniques: such as smoothing and shaping the wall; and (3) reactive techniques: such as actuators and microelectromechanical systems. However, due to complicated working conditions and serious environment, active techniques and reactive techniques are difficult to be applied in engineering practice, and passive technology is widely used in hypersonic vehicle design [14].

Passive techniques include a variety of technologies such as shaping and passive coatings [15-17]. There is a very effective method to arrange roughness on the wall. Roughness can be classified by many methods, such as two-dimensional roughness and three-dimensional roughness and isolated roughness and distributed roughness [18].

The effects of different types of roughness on the receptivity and stability of the boundary layer vary greatly. A large number of investigations have been carried out to investigate the effects of roughness on the receptivity and stability of the hypersonic boundary layer, and some achievements have been made [19-28]. Wang and Zhong and Fong et al. $[20,21]$ analyzed the effect of the height of isolated roughness on the receptivity of the hypersonic boundary layer by direct numerical simulation. They found that the height of isolated roughness made the disturbance grow instantaneously, and the growth rate increased with the increase of the height of isolated roughness. Zhao et al. [22] found that the transition position moved forward with the increase of the height of isolated roughness. Holloway and Sterett and Fujii [23, 24] found that the position of the isolated roughness would also affect the transition. Duan et al. [25, 26] found that the change of the position of isolated roughness directly affects the propagation of mode $S$ in the boundary layer by a direct numerical simulation method and then affected the receptivity mechanism of the hypersonic boundary layer.

In terms of isolated roughness, the research of distributed roughness is less $[18,27,28]$. Obviously, the effect of distributed roughness on the flow field is more complicated. Balakumar [18] used a numerical simulation method to analyze the influence of distributed roughness on the receptivity and stability of the hypersonic tip-cone flow boundary layer under slow acoustic disturbances, fast acoustic disturbances, and vortex wave. It was found that the second mode disturbance in the nose region was significantly suppressed. Desjouy et al. [27] discussed the effects of isolated and distributed roughnesses on the transition of the hypersonic boundary layer. They found that the transition of the boundary layer appeared more than that in the presence of a single roughness and the spacing between the two roughnesses has a great influence on the flow state in the boundary layer. Saric et al. [28] experimentally proved that the transition of the boundary layer can be effectively restrained by arranging the roughness array at the leading edge.

In this paper, a high-order accurate finite difference method is used to simulate the hypersonic flow field over the blunt wedge with a smooth wall and rough wall, and the effect of roughness on the flow state in the boundary layer is discussed. The shape of roughness is controlled by cubic polynomial, and the wall conditions are divided into a smooth wall and rough wall with isolated roughness and distributed roughness. The influence of roughness on the steady-state flow field is investigated firstly. On this basis, the influence of the roughness on the evolution process of disturbances in the boundary layer is analyzed by adding the slow acoustic disturbances to disturb the hypersonic flow field. The effect of roughness on evolution of different mode disturbances in the boundary layer is present by using the fast Fourier spectrum analysis (FFSA) method, and the influence mechanism of roughness on the receptivity of the hypersonic boundary layer is revealed.

\section{Governing Equations and Numerical Methods}

2.1. Governing Equations. A high-order finite difference method is employed to simulate the hypersonic flow field to analyze the evolution of the disturbance wave in the hypersonic boundary layer and to investigate the effect of roughness on receptivity of the boundary layer. The governing equations are two-dimensional conservation N-S equations which can be expressed as

$$
\frac{\partial \mathbf{U}}{\partial t}+\frac{\partial\left(\mathbf{F}-\mathbf{F}_{v}\right)}{\partial x}+\frac{\partial\left(\mathbf{G}-\mathbf{G}_{v}\right)}{\partial y}=0
$$

where $t$ is the time and $\mathbf{U}$ is the vector of conservative variables, $\mathbf{F}$ and $\mathbf{G}$ are the convective fluxes corresponding to $x$ and $y$ directions, respectively, and $\mathbf{F}_{v}$ and $\mathbf{G}_{v}$ are the viscous fluxes corresponding to the $x$ and $y$ directions, respectively. Their specific expressions are written as

$$
\begin{aligned}
\mathbf{U} & =(\rho, \rho u, \rho v, E)^{T}, \\
\mathbf{F} & =\left(\rho u, \rho u^{2}+p, \rho u v,(E+p) u\right)^{T}, \\
\mathbf{G} & =\left(\rho v, \rho u v, \rho v^{2}+p,(E+p) v\right)^{T}, \\
\mathbf{F}_{v} & =\left(0, \tau_{x x}, \tau_{x y}, K \frac{\partial T}{\partial x}+u \tau_{x x}+v \tau_{x y}\right)^{T}, \\
\mathbf{G}_{v} & =\left(0, \tau_{y x}, \tau_{y y}, K \frac{\partial T}{\partial y}+u \tau_{y x}+v \tau_{y y}\right)^{T},
\end{aligned}
$$

where $\rho, u, v, p, T$, and $K$ denote density, velocity along the $x$ axis and $y$ direction, pressure, temperature, and the heat transfer coefficient, respectively. $\tau$ and $E$ is the shear stress and total energy, respectively. The state equation for ideal gas is written as 


$$
P=\rho R T,
$$

where $R$ is $286.94 \mathrm{~J} /(\mathrm{kg} \cdot \mathrm{K})$.

In order to be convenient for computation, the governing equations are transformed from the physical coordinates $(x, y)$ to computational coordinates $(\xi, \eta)$, and the coordinate transformation coefficient is the Jacobian matrix $\mathbf{J}$ that can be expressed as

$$
\mathbf{J}=\frac{\partial(x, y)}{\partial(\xi, \eta)}=\left|\begin{array}{ll}
x_{\xi} & x_{\eta} \\
y_{\xi} & y_{\eta}
\end{array}\right|
$$

The governing equations in the computational coordinates are expressed as

$$
\frac{\partial \widehat{\mathbf{U}}}{\partial t}+\frac{\partial\left(\widehat{\mathbf{F}}-\widehat{\mathbf{F}}_{x}\right)}{\partial \xi}+\frac{\partial\left(\widehat{\mathbf{G}}-\widehat{\mathbf{G}}_{y}\right)}{\partial \eta}=0,
$$

where

$$
\begin{aligned}
\widehat{\mathbf{U}} & =J \mathbf{U}, \\
\widehat{\mathbf{F}} & =y_{\eta} \mathbf{F}-x_{\eta} \mathbf{G}, \\
\widehat{\mathbf{G}} & =-y_{\xi} \mathbf{F}+x_{\xi} \mathbf{G}, \\
\widehat{\mathbf{F}}_{v} & =y_{\eta} \mathbf{F}_{v}-x_{\eta} \mathbf{G}_{v}, \\
\widehat{\mathbf{G}}_{v} & =-y_{\xi} \mathbf{F}_{v}+x_{\xi} \mathbf{G}_{v} .
\end{aligned}
$$

2.2. Numerical Methods. In order to accurately simulate the hypersonic flow field over a blunt wedge and correctly capture the changes of the hypersonic flow field and the evolution of the disturbance wave in the boundary layer, a highorder finite difference method is used to directly simulate the hypersonic flow field. The governing equation can be decomposed into three parts for discretization. The convective flux is split by the Steger-Warming (S-W) splitting method into positive and negative flux terms, and then the fifth weighted essential nonoscillatory (WENO) scheme [29-31] is used to discretize the positive and negative flux terms. The central [25] difference method not only introduces unnecessary information but also causes calculation error. It is worse that they are not robust enough in the shock region, and there are very serious, nonphysical numerical oscillations generated in the shock region. So the upwind scheme is proper to discretize the convective flux, and there is a great advantage in this respect. The WENO scheme can effectively suppress the numerical oscillation in a discontinuous or large gradient region, such as the shock region, and the information of the flow field can be acquired stably and accurately. The physical information of the flow field is spread around caused by viscosity, so the viscous fluxes are discretized by a six-order central difference scheme. This method only introduces phase errors but not dissipative errors, which can ensure the accuracy of the calculation. The three-step third-order Runge-Kutta method is employed to advance time. This method is now widely used in the direct numerical simulation of hypersonic compressible flow fields, and the results achieved are good.

\section{Computational Condition}

A computational model is a hypersonic flow over a blunt wedge. Freestream conditions are adopted at the inlet, and extrapolation boundary conditions are adopted at the outlet; symmetric boundary conditions are employed at $y=0$ due to the symmetry of the model. The wall surface has no slip and no penetration and is isothermal. The influence of roughness on the receptivity of the hypersonic boundary layer is analyzed, and the wall surface conditions are divided into three types: wall surface without roughness, with isolated roughness, and with distributed roughness. The shape of roughness is controlled by a third-order polynomial, and the specific expression is as follows:

$$
\begin{aligned}
y_{n}(x)=\sum_{n}^{N}[ & \frac{R_{n}+h}{\cos \theta}+x \tan \theta-\frac{3 h}{\cos \theta}\left(\frac{x-x_{n}+h \sin \theta}{w \cos \theta+h \sin \theta}\right)^{2} \\
& \left.+\frac{2 h}{\cos \theta}\left(\frac{x-x_{n}+h \sin \theta}{w \cos \theta+h \sin \theta}\right)^{3}\right] \\
x_{n}=x_{0}+ & n R_{n} \cos \theta
\end{aligned}
$$

where $R_{n}, w, h$, and $\theta$ represent the nose radius, the half width of roughness, the height, and the half wedge angle, respectively, and $x_{n}$ is the center coordinate of roughness. $x_{0}=0.4109$. $N$ represents the number of roughness: when $N=1$, the wall surface condition is the wall with isolated roughness, and when $N>1$, it represents the wall with distributed roughness. For this paper, $N$ is 6 . The space between two roughnesses is $d=R_{n} \cdot \cos \theta=0.9962$. The calculational conditions and grid are given in Figure 1, where $\alpha$ represents the angle of attack. The subscripts " $\infty$ " and " $w$ " denote the freestream and wall condition, respectively. The geometric parameters of roughness are given in Table 1 . The number of meshes is $600 \times 150$, and the exponential stretching method is used to close meshes near the wall surface and head area properly where a strong shear flow is in the area.

Slow acoustic wave with single frequency is introduced as the disturbance wave to analyze the influence of slow acoustic disturbance on hypersonic flow field over the blunt wedge and flow structure in the boundary layer and to investigate the effect mechanism of roughness on the receptivity of the hypersonic boundary layer.

The steady flow field is computed first, and then the plus slow acoustic disturbances which can be expressed as (8) are continuously impinged on the upstream boundary after $t=100$ (dimensionless time), and the expression of slow acoustic is written as

$$
\left[\begin{array}{c}
u^{\prime} \\
v^{\prime} \\
p^{\prime} \\
\rho^{\prime}
\end{array}\right]=\left[\begin{array}{c}
\varepsilon \\
0 \\
-\frac{\varepsilon}{\mathrm{Ma}_{\infty}} \\
-\varepsilon \mathrm{Ma}_{\infty}
\end{array}\right] e^{i\left(k x-\left(F \cdot \operatorname{Re} / 10^{6}\right) t+\pi / 2\right),}
$$




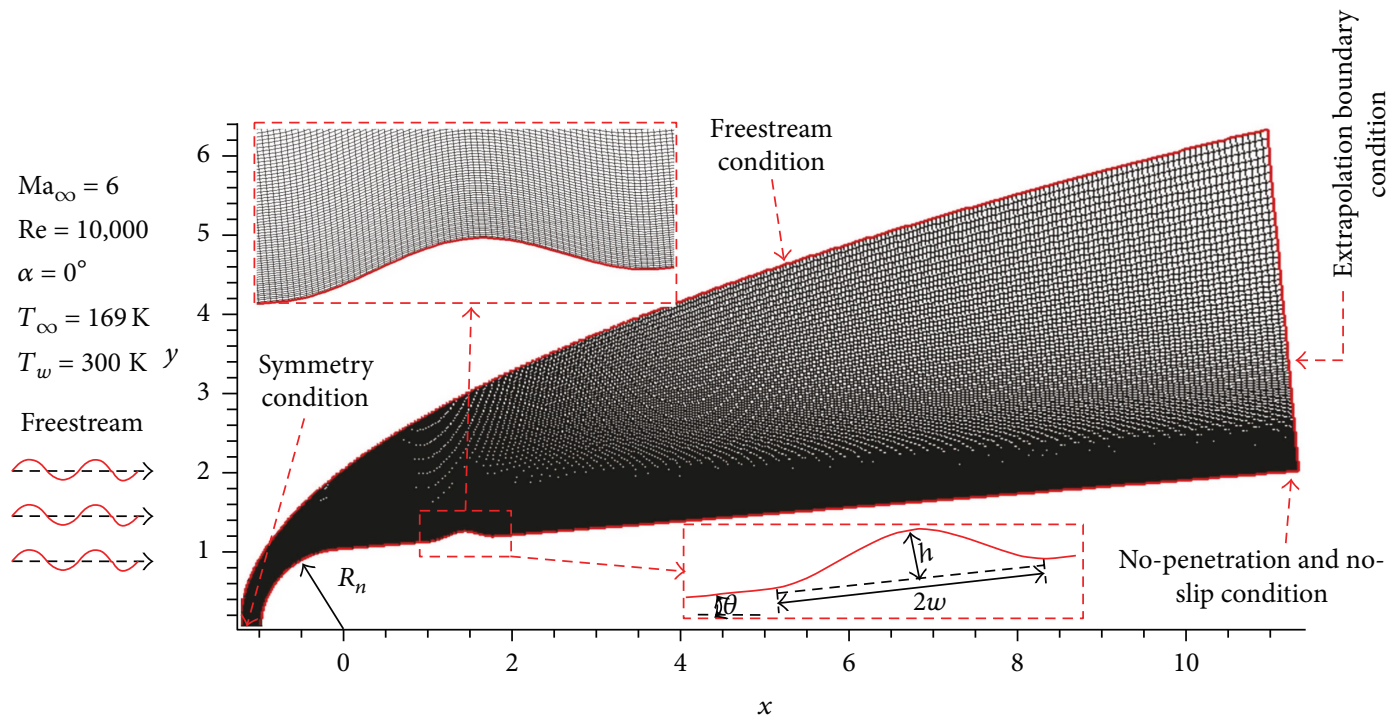

FIgURE 1: Calculational model and grid mesh.

TABLE 1: The condition of roughness.

\begin{tabular}{lccc}
\hline$R_{n}(\mathrm{~mm})$ & $\theta\left(^{\circ}\right)$ & $w(\mathrm{~mm})$ & $h(\mathrm{~mm})$ \\
\hline 1.0 & 5.0 & 0.40 & 0.10 \\
\hline
\end{tabular}

where the variable $\varepsilon$ is the amplitude $(\varepsilon=0.06), k$ is the wave number $\left(k=3.1446 \times 10^{-4}\right.$, and $F$ is the generalized frequency $(F=50 \pi)$ (dimensionless frequency $f=0.25$ ). The incident wave angle of acoustic disturbances is 0 . It should be noted that the superscript "' " represents the amount of disturbance in the flow field and the disturbance variable refers to the variable value of the unsteady flow field minus the variable value of the mean flow field at the same location. In this paper, each parameter is dimensionless, and flow parameter density $\rho$, velocity $u, v$, temperature $T$, and pressure $p$ are dimensionless by corresponding values $\rho_{\infty}, U_{\infty}$, $U_{\infty}, T_{\infty}$, and $\rho_{\infty} U_{\infty} U_{\infty}$, respectively. The head radius $R_{n}$ is chosen as the characteristic length; $x, y, w$, and $h$ are dimensionless; and the time $t$ is treated by $R_{n} / U_{\infty}$. The correctness of the code and the grid sensitivity are verified previously, which are not given here [32].

\section{Effect of Roughness on Mean Flow}

In order to accurately analyze the effect of roughness on the receptivity of the hypersonic boundary layer, a high-order finite difference method is used to directly simulate the hypersonic mean flow field over the blunt wedge in this section. Pressure contour and streamline pattern of the undisturbed hypersonic flow field are given in Figure 2, and Figure 2(a, b, c) corresponds to smooth wall, isolated roughness, and distributed roughness, respectively. It can be seen that the roughness has a great influence on the hypersonic flow field and the flow structure in the boundary layer. Compression wave-expression wave and compression wave are formed caused by isolated roughness. The temperature of flow increases when the flow crosses over the compression wave and some local high-temperature regions are formed, as shown in the figure. The intensity of the waves caused by roughness decreases downstream and dissipates gradually. The distributed roughness results in more compression and expansion waves in the flow field.

Flow separation is formed in the boundary layer-a vortex is formed at the leading edges of the isolated roughness, and five more long narrow vortices are formed between two roughnesses of the distributed roughness. Note that the flow lines above the roughness are almost parallel to the wall surface, and it indicates that the shear structure of the region above the roughness is substantially unaffected and the flow smoothly "slips" over the roughness region. Bountin et al. [14] studied the effect of distributed roughness on the undisturbed hypersonic flat boundary layer by means of experimental and numerical simulations. They found similar results as shown in Figure 3 that the flow structure of the strong shear layer above the roughness is not significantly altered while flow separations are formed in the boundary layer. Balakumar and Egorov et al. [33, 34] pointed out that the second mode acoustic disturbance may make the disturbance wave grow exponentially at the upstream and downstream of the separation region but remain neutral in the separation zone, so it is reasonable to believe that the wall surface condition can restrain the disturbance growth in the boundary layer and maintain the stability of the boundary layer to some extent.

Flow separation in the boundary layer can significantly change the mechanical and thermodynamic mechanisms in the boundary layer; Hirschel [35] pointed out that the change of the mechanical and thermodynamic mechanisms in the boundary layer has a great influence on the stability of the boundary layer. The distribution of wall pressure, friction coefficient, and heat flux along the flow direction are given in Figures 4(a), 5, and 6, respectively, and Balakumar's results are given in Figures 4(b) and 4(c) where part of the calculation parameters is given. Figures 4(b) and 4(c) are the results corresponding to isolated roughness and disturbance 


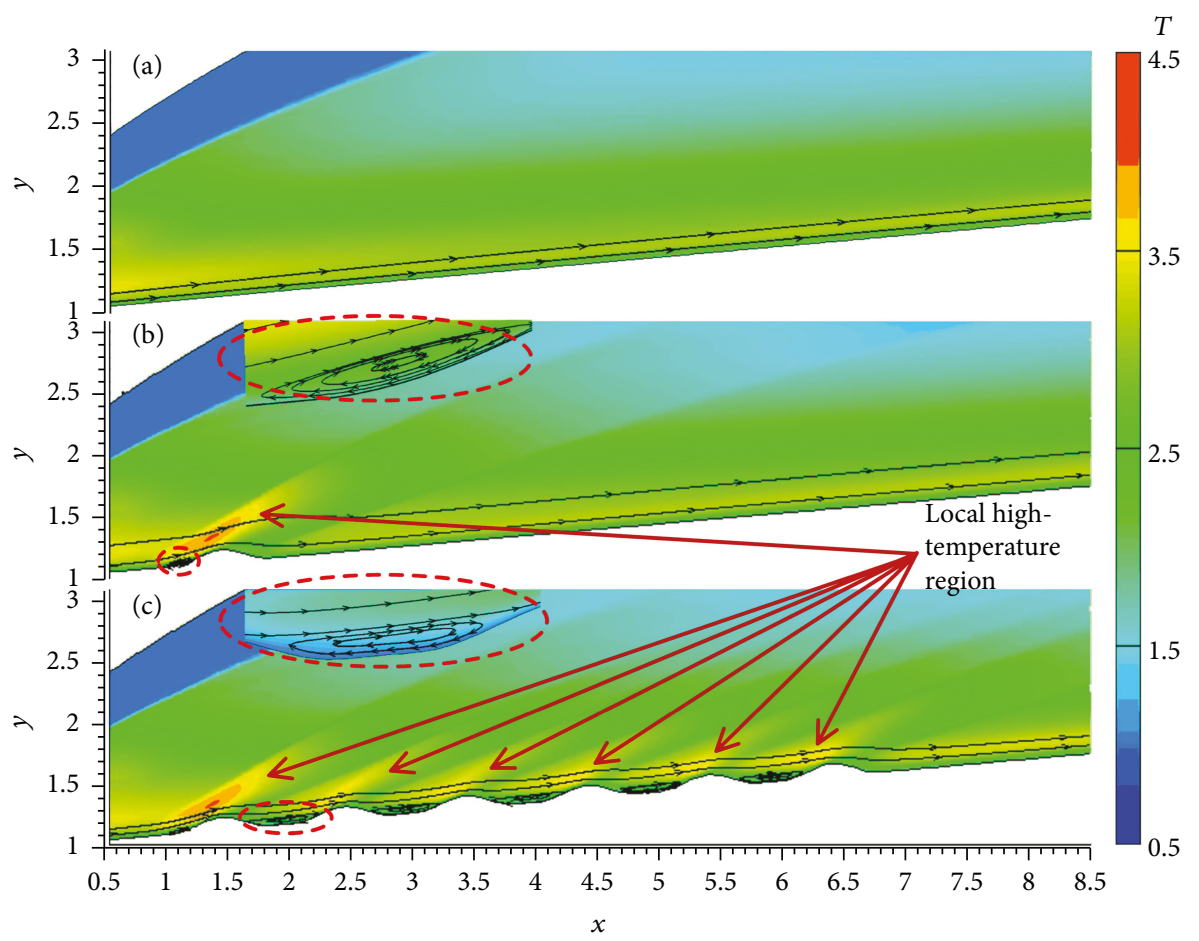

Figure 2: Pressure contour and streamline pattern of the undisturbed hypersonic flow field.

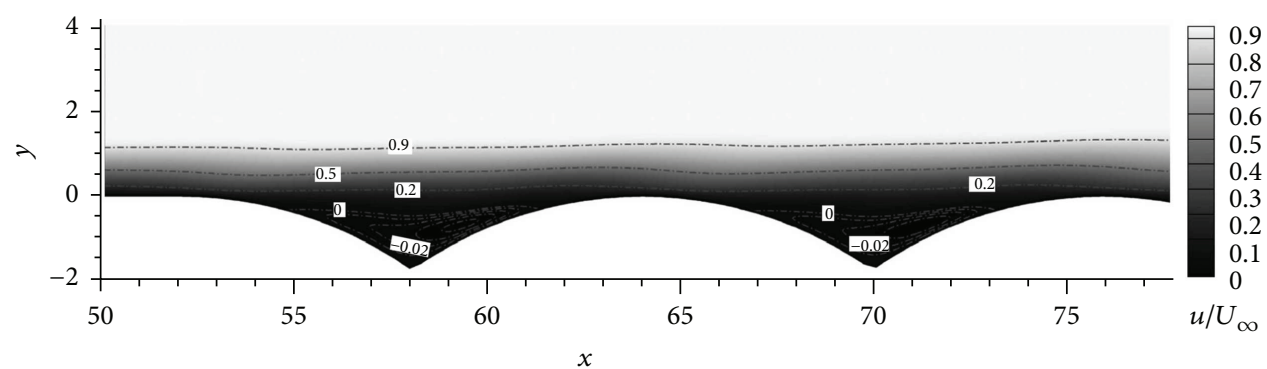

FIGURE 3: Velocity along the $x$-axis of steady flow in the boundary layer in literature [14].

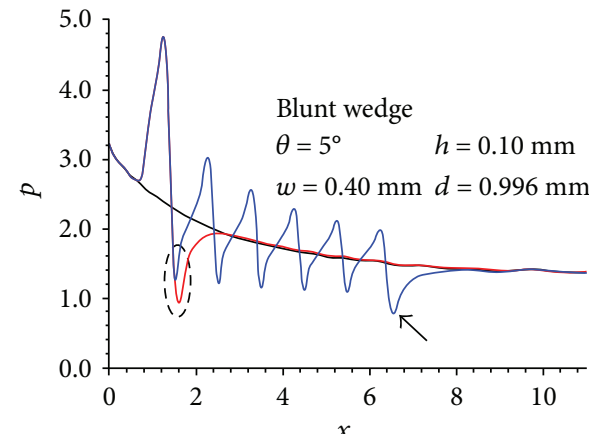

_ Smooth wall

- Isolated roughness

- Distributed roughness

(a)

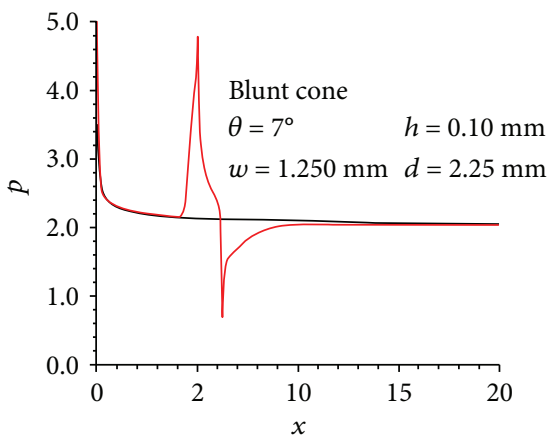

Smooth wall
- Isolated roughness

(b)

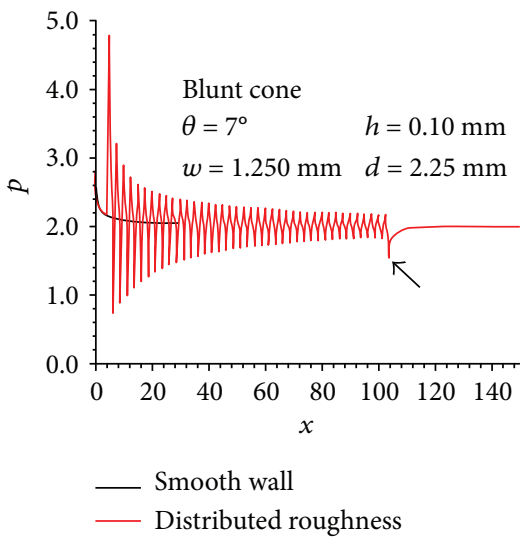

(c)

Figure 4: Comparison of distribution of wall pressure along the $x$-axis with the literature [36]. 


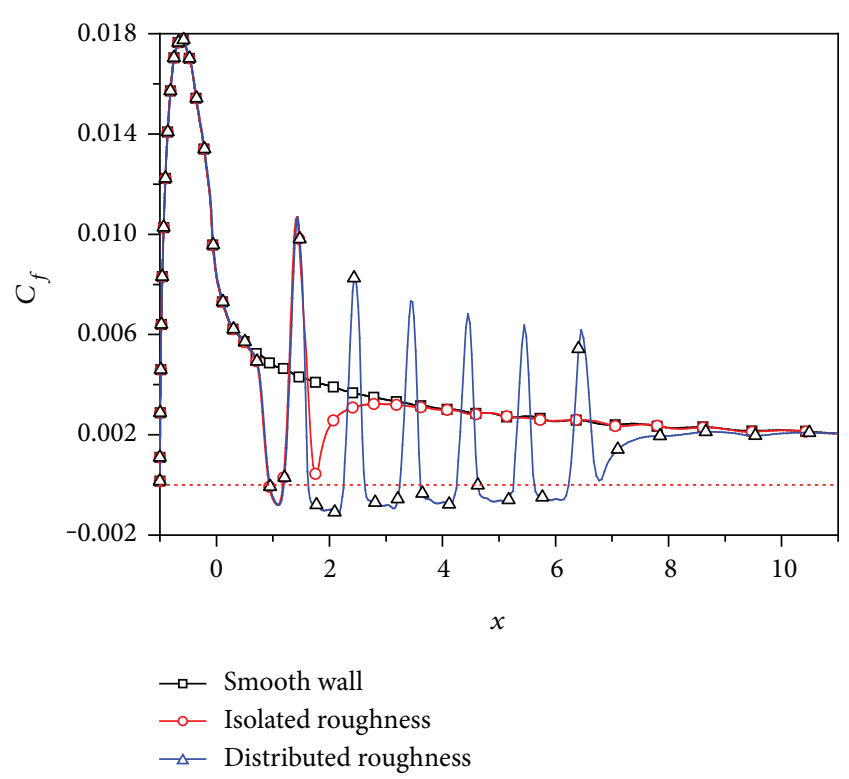

FIgURE 5: The distribution of friction coefficient of the wall along the flow distribution.

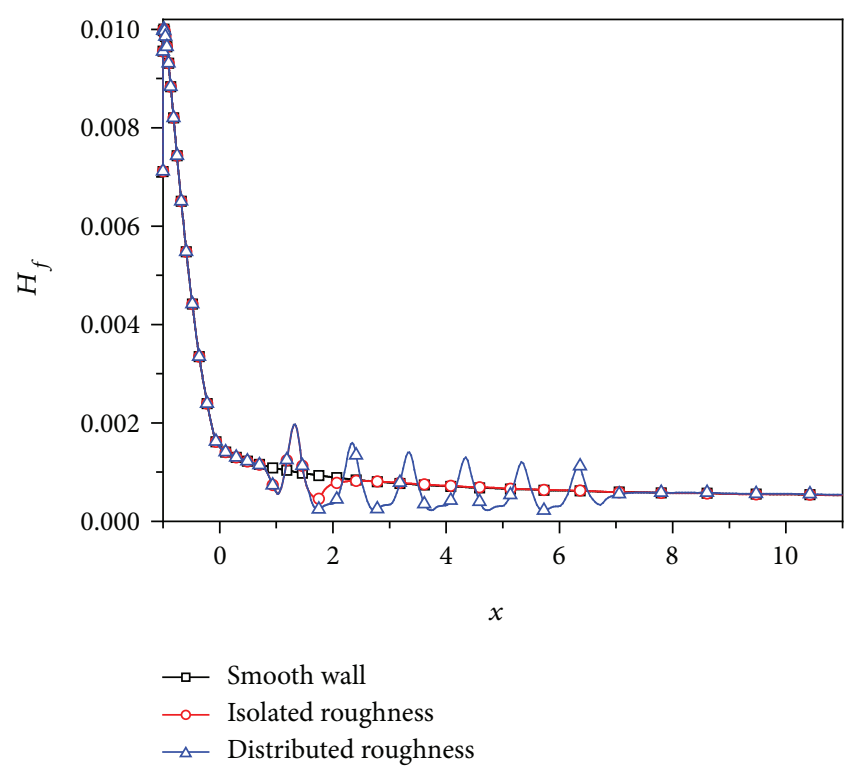

Figure 6: The distribution of heat flux of the wall along the flow direction.

roughness $(N=40)$, respectively. It can be seen from the figures that flow structure is significantly changed by the roughness in the boundary layer, and the trend of wall pressure under the two wall conditions is basically the same. As flow in the boundary layer evolves over each roughness, wall pressure increases, then decreases, and finally recovers to the undisturbed state. There is a strong expansion-compression process in the trailing edge region of the last roughness, as indicated by the arrows in the figures. It indicates that there is a strong adverse pressure gradient in this region, which may even result in flow separation. However, there also are some differences between the two kinds of roughness. In this paper, the position and magnitude of the minimum pressure
TABLE 2: Integral value of friction coefficient and heat flux along the wall.

\begin{tabular}{lccc}
\hline & Smooth wall & Isolated roughness & Distributed roughness \\
\hline $\int C_{f} d s$ & 0.05488 & 0.05249 & 0.04639 \\
$\int H_{f} d s$ & 0.01822 & 0.01817 & 0.01771 \\
\hline
\end{tabular}

of the isolated and distributed roughnesses are different, but this phenomenon does not exist in the results of Balakumar [33]. The reason for this phenomenon is a long and narrow vortex formed between the two roughnesses in this paper and that the incoming flow meeting the vortex in this region results in an increase in pressure.

In order to analyze the influence of the roughness on the strong shear flow in the boundary layer and the influence of the incoming flow on the wall heating, Figures 5 and 6 demonstrate the distribution of the friction coefficient and the heat flux of the wall, respectively. The friction coefficient of the wall decreases significantly in the leading edge and the trailing edge of the roughness and reaches the maximum value in the center of the roughness, and the reason for this phenomenon is the change of boundary layer thickness. The friction coefficient is negative in some areas because of the existence of vortices in these areas. So friction drag may reduce to a certain extent caused by cortices. Similarly, the heat flux also decreases significantly in these areas. It shows that the vortex in the hypersonic boundary layer can reduce friction drag and restrain the heating of the wall to a certain extent.

The integral values of the friction coefficient and heat flux along the wall are given in Table 2. It can be seen that the friction drag of the wall and the total heat transfer of the distributed roughness are reduced by $15.47 \%$ and $2.80 \%$, respectively, compared with those of the smooth wall. These values are similar to Bountin et al.'s [14] results, which show that distributed roughness reduces the friction drag of the hypersonic flat boundary layer by $17.64 \%$. Through the above analysis, it can be found that arranging the distributed roughness on the wall can achieve the purpose of reducing drag and heat to a certain extent, while not significantly damaging the strong shear flow above the roughness.

\section{Effect of Roughness on the Unsteady Flow Field}

It has been pointed out above that in the steady flow field, arranging distributed roughness on the wall can reduce friction drag and total heat transfer to a certain extent. But the hypersonic vehicle inevitably encounters acoustic disturbance during the flight [37]. In this paper, a slow acoustic wave with single frequency is introduced as the disturbance and the effect mechanism of roughness on the receptivity of the boundary layer under the action of slow acoustic disturbance is revealed. The contours of velocity along the $y$-axis under different wall conditions are shown in Figure 7, and Figures $7(\mathrm{a})-7(\mathrm{c})$ correspond to smooth wall, isolated 


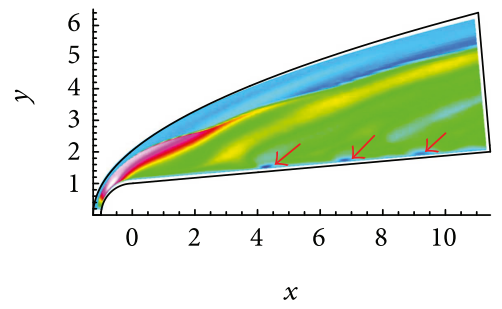

(a) Small wall

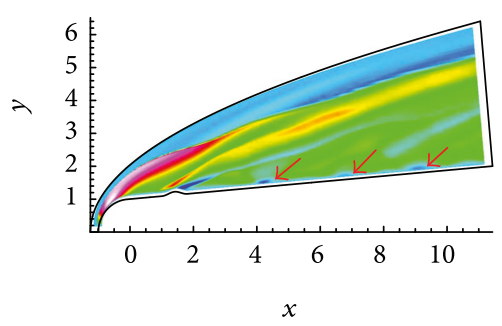

(b) Isolated roughness

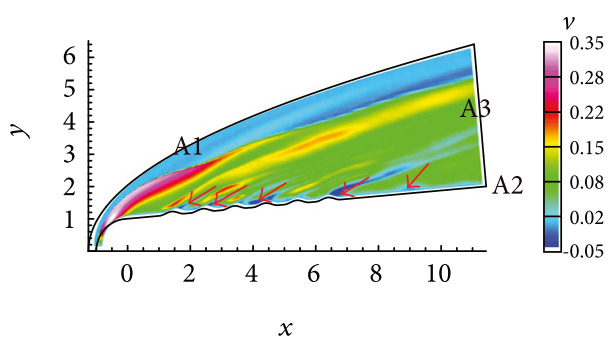

(c) Distributed roughness

FIgURE 7: Contours of velocity along the $y$-axis under different walls.

roughness, and distributed roughness, respectively. It can be seen that the hypersonic flow field under slow acoustic disturbance is mainly divided into three regions: Region A1-the interaction between slow acoustic disturbance and shock wave leads to the shock wave deformation and the formation of a local high-velocity region that is basically unaffected by wall conditions. Region A2-the interaction between a slow acoustic disturbance and the boundary layer results in flow separation in the boundary layer, as shown in the figure. That more separation regions are formed in the boundary layer caused by distributed roughness may be inhibited by the heating of the wall by the flow. Region A3-the region between Regions A1 and A2, relatively speaking, is minimally affected by slow acoustic disturbance, but when distributed roughnesses are arranged on the wall, the region becomes more complex under the influence of slow acoustic disturbance. The hypersonic flow field of Region A3 is significantly changed by the compression and expansion waves induced by the distributed roughness, especially the first compression wave and the last two expansioncompression waves. The interaction between the acoustic disturbance and hypersonic flow field over the sharp cone is studied by Kara [36], and the author found that Region A3 is very quiet. So the author considered that acoustic waves are weakly transmitted through shock. There is a difference between the two results, and we believe that the reason for this difference is the amplitude of disturbance while the amplitude of acoustic waves in literature is $2 \times 10^{-5}$. It should be noticed that an inhomogeneity of perturbations is formed near the shock region. The inhomogeneity is caused by the interaction between the freestream disturbances and shock. The freestream disturbances go through the shock and result in the deformation of shock. The deformation of shock causes some tiny perturbations formed near the shock region. Thus, there is an inhomogeneity of perturbations in the oncoming flow. In addition, the great deformation of shock near the nose of the blunt wedge also affects the oncoming flow in the downstream region.

The variation of flow structure in the hypersonic flow field, especially in the boundary layer, has a great influence on the evolution of acoustic disturbance in the boundary layer [38]. Therefore, it should be believed that the wall conditions have a great influence on the receptivity mechanism of the hypersonic boundary layer. The instantaneous pressure disturbance in the boundary layer for acoustic disturbance at different stations is shown in Figure 8. It is worth emphasizing that the magnitude of acoustic disturbance is two to three orders higher than that of acoustic disturbance in freestream and is four to five orders higher than the results of Balakumar [18], even higher. This is because the magnitude of acoustic disturbance is magnified when acoustic waves are transmitted through the shock wave. In this paper, the influence of roughness on the flow in the boundary layer is more concerning, and the influence of isolated and distributed roughnesses on the receptivity is analyzed. Therefore, several stations along the flow direction are selected for analysis: (1) the stations around the isolated roughness or the first roughness of the distributed roughness $(x=$ 1.00427 1.793163, 5 stations), (2) the station between two roughnesses $(x=1.897203)$, (3) downstream region of the first roughness or around the second roughness $(x=$ 2.203272 2.606049, 3 stations), and (4) the downstream of the distributed roughness $(\mathrm{x}=7.798937-9.719503,3$ points $)$.

Obviously, the magnitudes of the disturbance wave at different stations under the action of a slow acoustic disturbance obviously change cyclically, and the boundary layer is disturbed by the slow acoustic wave more lately at the downstream station. The evolution of disturbance waves in the boundary layer can simply be divided into two stages: stages $\mathrm{T} 1$ and T2, as shown in Figure 8. At stage T1 is the response phase when the disturbance wave starts to affect the flow in the boundary layer and the pressure begins to change. The evolution of disturbance waves is not periodic, and the amplitude of the disturbance wave is small at this stage. At stage T2, the flow field is continuously and steadily affected by the slow acoustic disturbance, and the evolution of disturbance waves is periodic. The wall condition remarkably changes the evolution of the slow acoustic disturbance, and the magnitude of the acoustic disturbance becomes bigger at this stage.

After the acoustic disturbance enters the flow field through the bow shock wave, the disturbance propagates to the upstream-half region of roughness, $(x=1.001427$ 1.402937). The magnitude of disturbance waves increases, and the growth of disturbance is promoted by roughness in the boundary layer. Growth rate increases with the increase of wall curvature. For the downstream-half region of the roughness $(x=1.402937 \sim 1.793163)$, the magnitude of disturbance waves decreases. The growth of acoustic disturbance in the boundary layer is suppressed in this region and is suppressed with the increase of curvature. It should be noted that the magnitude of the disturbance 

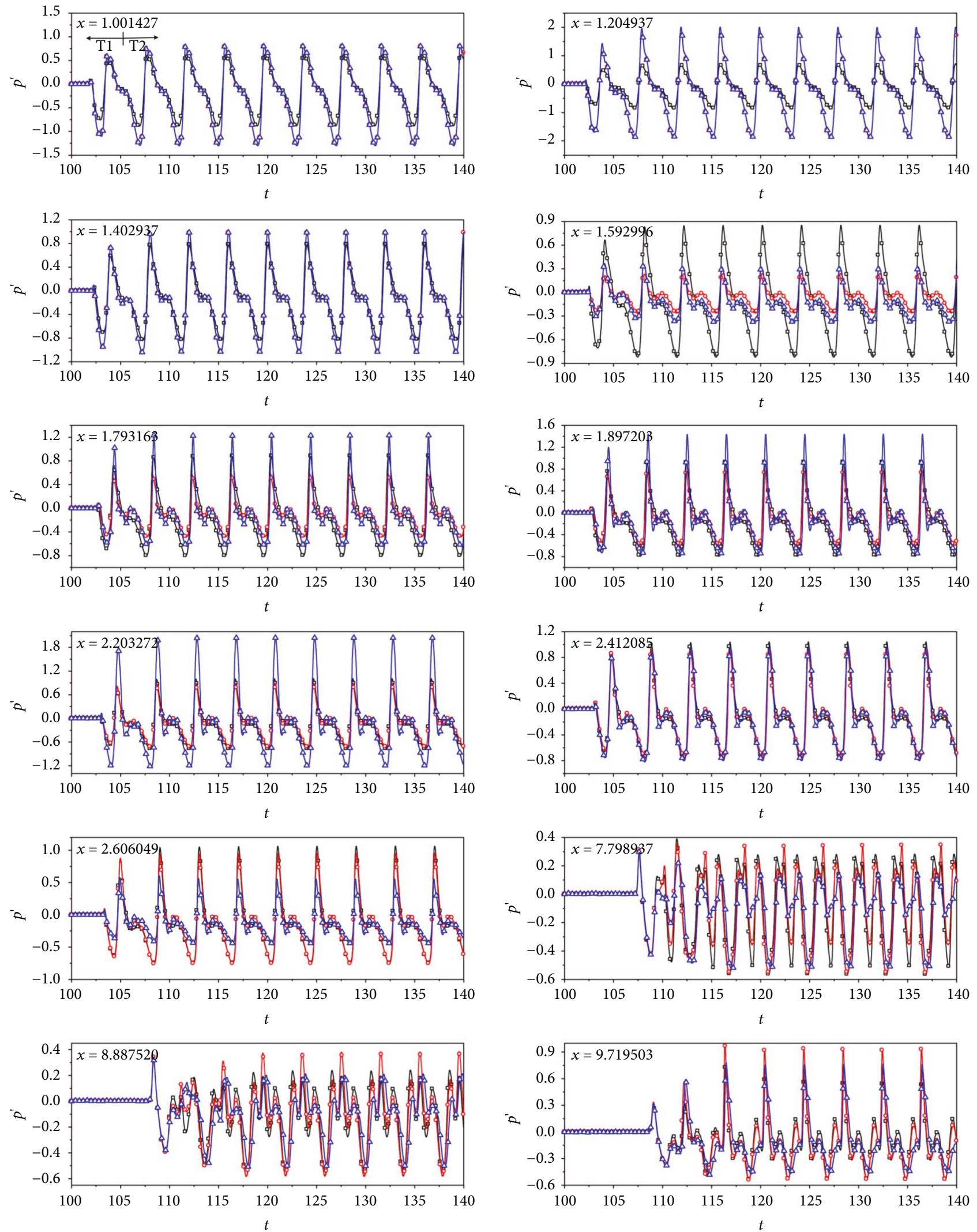

$\rightarrow-$ Smooth wall

- Isolated roughness

$\triangle$ Distributed roughness

FIgURE 8: Pressure disturbance on the wall at different stations along the flow direction. 


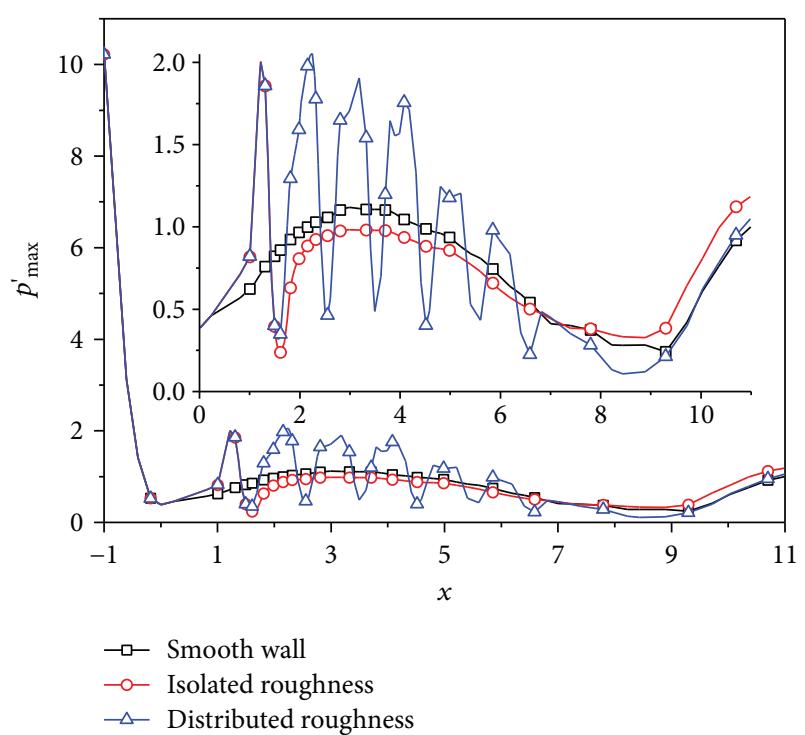

FIGURE 9: The distribution of maximum pressure disturbances along the flow direction.

wave corresponds to distributed roughness increases at the station $(x=1.793163)$. That indicates that the evolution of the acoustic disturbance at this station is affected by the downstream roughness or the vortex between two roughnesses. Flow is compressed at this position, and the magnitude of the acoustic disturbance increases. At $x=1.897203$, the magnitude of disturbance waves corresponds to the isolated roughness which continues to decrease, but the magnitude of the disturbance wave corresponds to distributed roughness increases. It is worth noting that at this point, the pressure of the wall with distributed roughness or without roughness is the same in the undisturbed flow field. It indicates that the vortex amplifies the magnitude of the acoustic disturbance in the boundary layer. In the range of $x=2.203272$ to 2.606049 , the magnitude of the disturbance waves for isolated roughness decreases continuously. The magnitude variation trend of pressure disturbance of the distributed roughness is the same as that of the last upstream roughness which shows the process of enlargementreduction during flow over the roughness. The magnitude of disturbance waves for distributed roughness is the same as that of the smooth wall at the center of roughness. The evolution of disturbance waves in the downstream region of the distributed roughness in the boundary layer is more concerning. In the range of $x=7.798937$ to 9.719503 , the acoustic disturbance becomes more complicated. It indicates that the modes of the disturbance waves in the boundary layer increase and there is the existence of competition between different mode disturbances in the boundary layer. The modal analysis of the acoustic disturbance in the boundary layer will be given in the next section. At $x=7.798937$ and 8.887520 , the variation of disturbance wave magnitude of the distributed roughness is small, and the growth of the disturbance wave is restrained. At $x=9.719503$, the restraining effect weakens, and the magnitude of the disturbance wave for the distributed roughness approximates that of the smooth wall.
It is worth noting that in the range of $x=7.798937$ to 9.719503, the magnitude of the disturbance waves for the isolated roughness is always high. That is to say, the disturbance waves for the isolated roughness are firstly restrained, then grow, and gradually exceed those for the smooth wall and distributed roughness.

Figure 9 shows the distribution of the maximum values of the magnitude of disturbance waves at different wall conditions along the flow direction. It can be seen that the acoustic disturbance is the magnitude in the upstream-half region of roughness and suppressed in the downstream-half region, and the intensity of amplification or inhibition is enhanced with the increase or decrease of the wall curvature.

In addition, the vortex also amplifies the acoustic disturbance in the boundary layer, which is the strongest at the center of the vortex.

\section{Analysis of Disturbance Wave Modes in Boundary Layers}

In order to reveal the influence of roughness on the receptivity of the boundary layer clearly, the evolution process of acoustic disturbances with different modes in the boundary layer is analyzed.

Fast Fourier spectrum analysis (FFSA) is used to decompose the time domain signal in the boundary layer and expand it in the frequency domain to get the evolution process of the acoustic disturbance with different frequencies. This method is widely used to analyze receptivity of the hypersonic boundary layer with roughness under freestream disturbances, and satisfactory results are attained [21, 39]. The Fourier transform formula is as follows:

$$
p^{\prime}(x, y, t)=\operatorname{Re}\left(\sum_{n=0}^{N}\left|p_{n}^{\prime}(x, y)\right| e^{i[-n \omega t+\varphi n(x, y)]}\right)
$$

Figure 10 shows the FFSA amplitude-frequency diagram at different stations, and it shows that under the slow acoustic wave with single frequency, the dominant modes in the boundary layer are fundamental frequency and harmonics while the other modes can be insignificant. In the region of $x=1.402937$ to 1.793163 , the fundamental frequency (frequency of the fundamental frequency mode $f$ is 0.25 , frequency of the second-order harmonic disturbance $f$ is 0.50 , frequency of the third-order harmonic disturbance $f$ is 0.75 , etc.) and each harmonic order mode are amplified in the upstream-half region of roughness. The dominant mode in the boundary layer is taken as the fundamental frequency mode under the three wall conditions, and the proportion of each order harmonic mode decreases with the increase of frequency. The evolution of fundamental and each order harmonic mode is restrained in the downstream-half region of the roughness. For the smooth wall, the fundamental mode is still the dominant mode, but for the rough wall, the dominant mode becomes the secondorder harmonic mode. That is to say, in this region, the roughness can restrain the fundamental mode better than the second-order harmonic. 

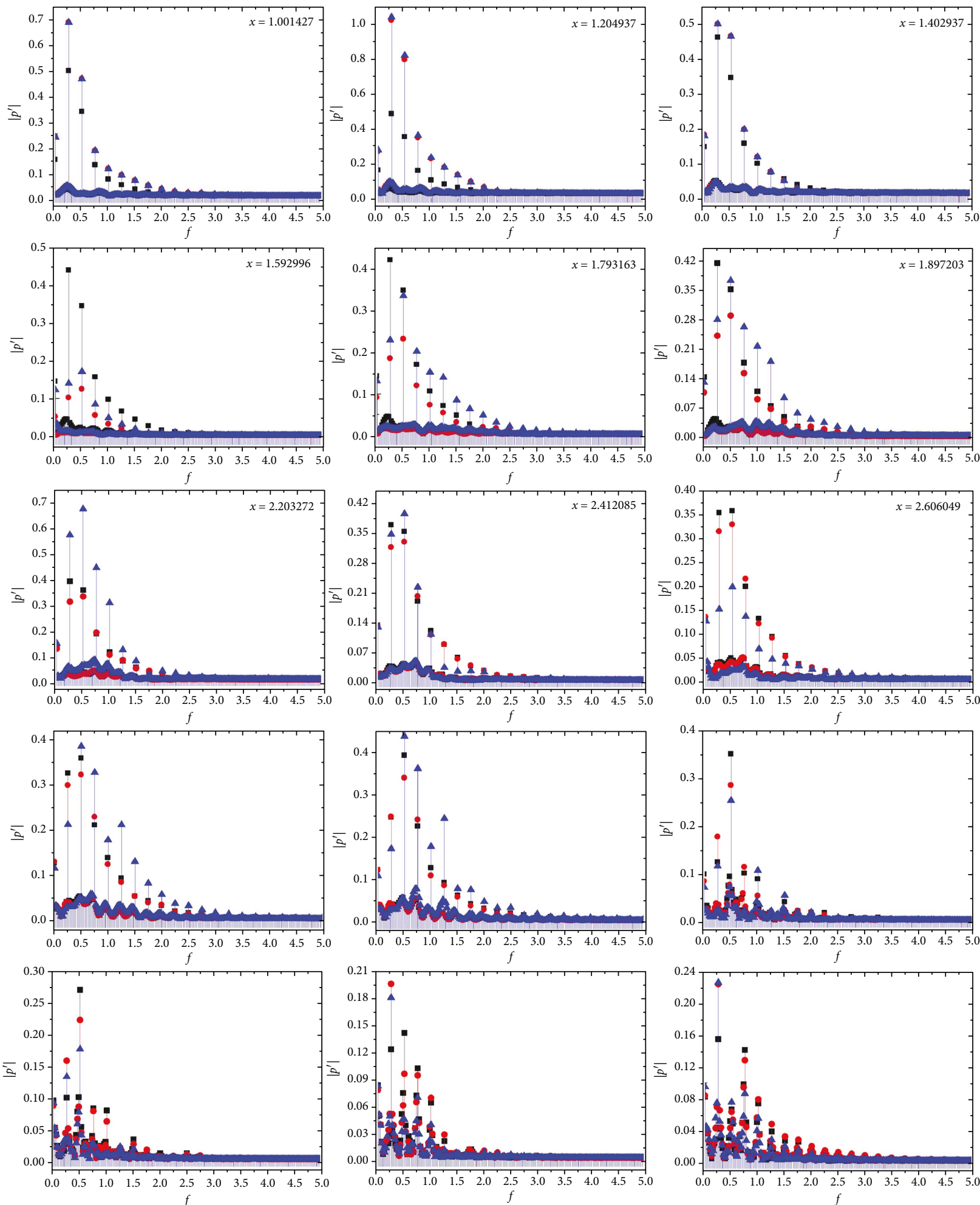

Smooth wall
Isolated roughness
Distributed roughness

FIgURE 10: Comparison of Fourier frequency spectral analysis of pressure disturbance in the boundary layer for different wall conditions. 

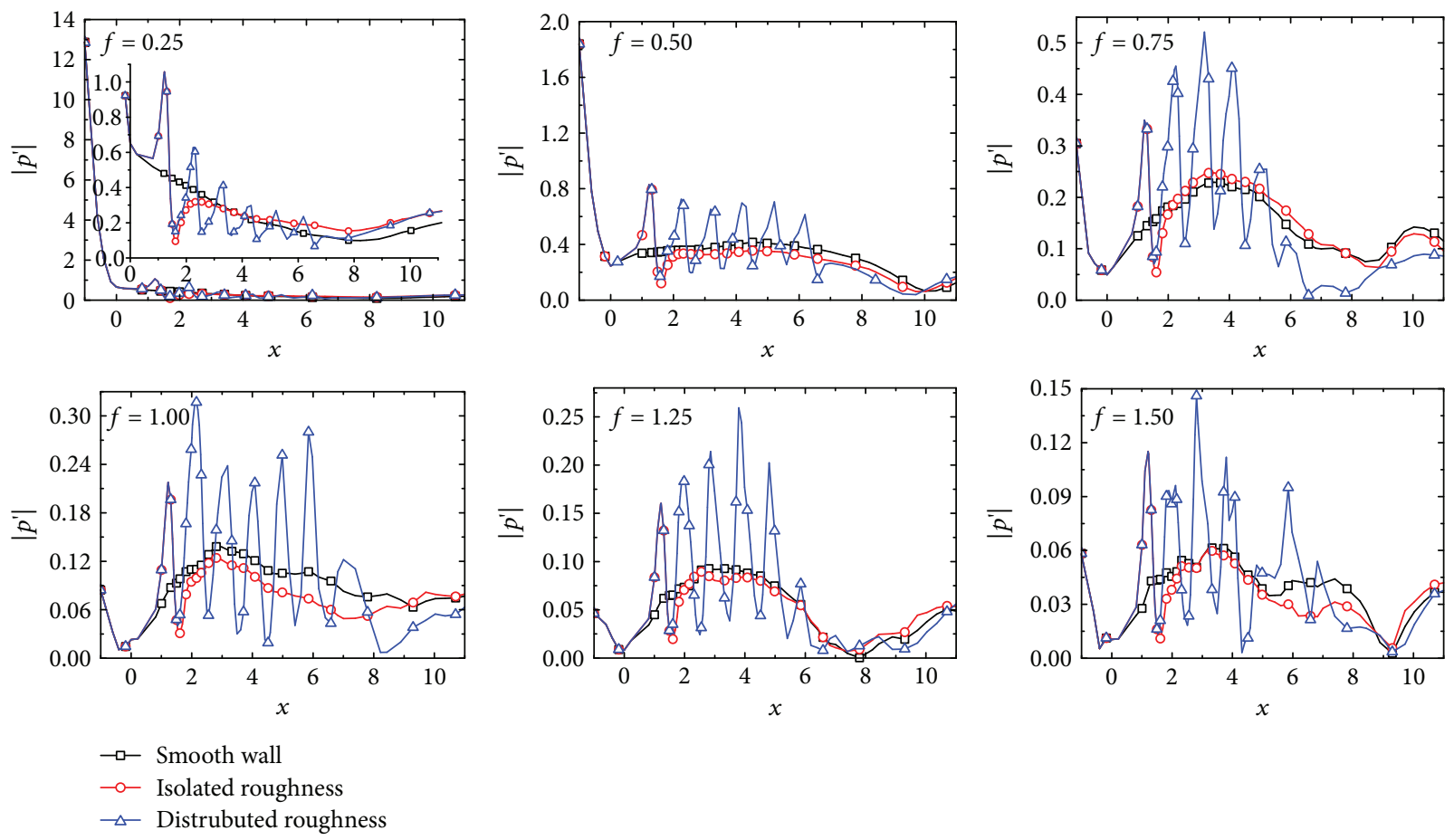

$\multimap$ Isolated roughness
$\longleftarrow$ Distrubuted roughness

FIGURE 11: Comparison of evolution of different modes along the wall for different wall conditions.

At $x=1.897203$, the FFSA magnitude of the fundamental mode and each order harmonic mode of the isolated roughness are smaller than those of the smooth wall and distributed roughness wall, and the FFSA magnitude of each order harmonic disturbance for the distributed roughness is larger than that of the smooth wall except the fundamental mode. It implies that for the distributed roughness, the fundamental mode is suppressed in the vortex region, but the evolution of each order harmonic mode is promoted. The same phenomenon occurs at the next two vortices $(x=2.860089$ and 3.891759). In the region of $x=2.203272$ to 2.606049, the trends of the fundamental mode and harmonic mode of each order are similar to those of the smooth wall but smaller except for the third-order harmonic mode at $x=$ 2.606049 . It is noteworthy that at $x=2.606049$, the dominant mode for the smooth wall transforms from the fundamental mode to the second-order harmonic mode. The fundamental mode and harmonic mode for the distributed roughness are suppressed and then amplified in this region. The dominant mode is always the second-order harmonic frequency mode, but its proportion is decreasing gradually, while the proportion of the harmonic mode of each order is increasing gradually.

For the downstream region of distributed roughness, the second-order harmonic mode is still the dominant mode in the range of $x=6.817512$ to 7.798937 under different wall conditions, but the magnitude of the distributed roughness is significantly smaller than that of the other two types of wall conditions; even the third-order and fifth-order harmonic modes tend to disappear. That is to say, the evolution of the acoustic disturbances in the boundary layer is obviously inhibited in the downstream region of the distributed roughness, which does not last for a long distance. At the station of $x=8.888520$ and 9.719503 , the FFSA magnitude of the acoustic disturbances of the distributed roughness gradually increases, especially the fundamental mode, which rapidly increases and becomes the dominant mode in the boundary layer.

Figure 11 shows the distribution of the fundamental mode and harmonic modes from the second order to the sixth order in the boundary layer along the flow direction. It can be seen that the fundamental frequency and each order harmonic mode decay rapidly in the head region. The degree of attenuation of different modes becomes greater with the frequency decrease.

The variation trend for different wall conditions on the fundamental mode and harmonic mode is basically the same, but there are still some differences. In general, different modes are amplified in the upstream-half region of the roughness and suppressed in the downstream-half region of the roughness, and this suppression will last for a distance. The different modes are suppressed for different distances; some disturbance modes are suppressed for a long distance, while others are short.

For the isolated roughness, the second-order harmonic, fourth-order harmonic, and sixth-order harmonic modes in the boundary layer are suppressed for a long distance while the fundamental mode and the thirdorder harmonic mode are suppressed by isolated roughness for a short distance in the boundary layer. Especially for the fundamental mode, the fundamental mode corresponding to the isolated roughness is always higher than that for the smooth wall after the inhibition effect of roughness is not effective. 
In the downstream region of the distributed roughness, the harmonic modes of each order are suppressed, but the increase of the fundamental mode in the boundary layer is not inhibited. The fundamental mode begins to grow rapidly after passing through the distributed roughness.

\section{Conclusions}

A high-order accurate finite difference method is used to simulate the hypersonic flow field over a blunt wedge with isolated roughness and distributed roughness or without roughness. The effect of roughness on steady and unsteady hypersonic flow fields is analyzed. The effect of roughness on the evolution of disturbance waves caused by a slow acoustic wave in the boundary layer is discussed by fast Fourier spectrum analysis (FFSA) method, and some conclusions are drawn.

(1) Compared to a smooth wall, distributed roughness arranged on the wall can reduce friction drag and total heat transfer to a certain extent, while the strong shear flow above the roughnesses does not significantly damage.

(2) The amplitude of disturbance waves under the fundamental mode and the harmonic mode of each order increases markedly in the upstream-half region of roughness while decreases markedly in the downstream-half region of roughness. Disturbance waves are suppressed in the downstream region of isolated and distributed roughnesses for a distance. Some disturbance modes are suppressed for a long distance, while others are short.

(3) The mode competition always exits among different modes both in the temporal domain and in the frequency domain in the boundary layer. The dominant mode is changed by roughness: the fundamental mode is the dominant mode until $x=2.606049$ for a smooth wall in the boundary layer while the dominant mode is changed to the second-order harmonic mode at $x=1.592996$ for a rough wall.

(4) The evolution of disturbance waves in the boundary layer is affected by the vortices. The vortex amplifies the magnitude of the acoustic disturbance in the boundary layer: the fundamental mode is suppressed in the vortex region, but the evolution of each order harmonic mode is promoted.

\section{Conflicts of Interest}

The authors declare that there is no conflict of interests regarding the publication of this paper.

\section{Acknowledgments}

The authors gratefully acknowledge the financial support of the National Natural Science Foundation of China (Grant nos. 11472086 and 11532013).

\section{References}

[1] S. P. Schneider, "Flight data for boundary-layer transition at hypersonic and supersonic speeds," Journal of Spacecrafts and Rockets, vol. 36, no. 1, pp. 8-20, 1999.

[2] S. P. Schneider, "Hypersonic laminar-turbulent transition on circular cones and scramjet forebodies," Progress in Aerospace Sciences, vol. 40, no. 1-2, pp. 1-50, 2004.

[3] P. J. P. Jeroen, V. D. Eynde, and N. D. Sandham, "Numerical simulations of transition due to isolated roughness elements at Mach 6," AIAA Journal, vol. 54, no. 1, pp. 53-65, 2016.

[4] Y. B. Ma and X. L. Zhong, "Receptivity of a supersonic boundary layer over a flat plate. Part 2 . Receptivity to freestream sound," Journal of Fluid Mechanics, vol. 488, pp. 79121, 2003.

[5] Y. B. Ma and X. L. Zhong, "Receptivity of a supersonic boundary layer over a flat plate. Part 3. Effects of different types of free-stream disturbances," Journal of Fluid Mechanics, vol. 532, pp. 63-109, 2005.

[6] P. Balakumar and M. A. Kegerise, "Receptivity of hypersonic boundary layers over straight and flared cones," AIAA Journal, vol. 53, no. 8, pp. 2097-2109, 2015.

[7] A. Cerminara and N. D. Sandham, "Acoustic leadingedge receptivity for supersonic/hypersonic flows over a blunt wedge," AIAA Journal, vol. 55, no. 12, pp. 4234-4244, 2017.

[8] D. Park and S. O. Park, "Influence of two-dimensional smooth humps on linear and non-linear instability of a supersonic boundary layer," Computers \& Fluids, vol. 79, pp. 140-149, 2013.

[9] M. Olaf, I. Gianluca, and E. S. G. Shaqfeh, "Nonlinear instability of a supersonic boundary layer with two-dimensional roughness," Journal of Fluid Mechanics, vol. 752, pp. 497$520,2014$.

[10] F. Qin and X. Wu, "Response and receptivity of the hypersonic boundary layer past a wedge to free-stream acoustic, vortical and entropy disturbances," Journal of Fluid Mechanics, vol. 797, pp. 874-915, 2016.

[11] Y. D. Zhang, D. X. Fu, Y. W. Ma, and X. L. Li, "Receptivity to free-stream disturbance waves for hypersonic flow over a blunt cone," Science in China Series G: Physics, Mechanics and Astronomy, vol. 51, no. 11, pp. 1682-1690, 2008.

[12] R. L. Kimmel, "Aspects of hypersonic boundary layer transition control," in 41st Aerospace Sciences Meeting and Exhibit, Reno, NV, USA, January 2003.

[13] L. W. Chen, G. L. Wang, and X. Y. Lu, "Numerical investigation of a jet from a blunt body opposing a supersonic flow," Journal of Fluid Mechanics, vol. 684, no. 11, pp. 85110, 2011.

[14] D. Bountin, T. Chimitov, A. Maslov, A. Novikov, I. Egorov, and F. Alexander, "Stabilization of a hypersonic boundary layer using a wavy surface," AIAA Journal, vol. 51, no. 5, pp. 1203-1210, 2013.

[15] A. V. Fedorov, N. D. Malmuth, A. Rasheed, and H. G. Hornung, "Stabilization of hypersonic boundary layers by porous coatings," AIAA Journal, vol. 39, no. 4, pp. 605-610, 2001.

[16] A. Rasheed, H. G. Hornung, A. V. Fedorov, and N. D. Malmuth, "Experiments on passive hypervelocity boundary layer control using an ultrasonically absorptive surface," AIAA Journal, vol. 40, no. 3, pp. 481-489, 2002. 
[17] N. D. Sandham and H. Lüdeke, "Numerical study of Mach 6 boundary-layer stabilization by means of a porous surface," AIAA Journal, vol. 47, no. 9, pp. 2243-2252, 2009.

[18] P. Balakumar, "Receptivity of hypersonic boundary layers to distributed roughness and acoustic disturbances," in 51st AIAA Aerospace Sciences Meeting including the New Horizons Forum and Aerospace Exposition, Dallas, TX, USA, January 2012.

[19] A. Sescu and D. Thompson, "On the excitation of Görtler vortices by distributed roughness elements," Theoretical and Computational Fluid Dynamics, vol. 29, no. 1-2, pp. 67-92, 2015.

[20] X. Wang and X. Zhong, "Receptivity of a hypersonic flat-plate boundary layer to three-dimensional surface roughness," Journal of Spacecraft and Rockets, vol. 45, no. 6, pp. 1165-1175, 2008.

[21] K. D. Fong, X. Wang, and X. Zhong, "Numerical simulation of roughness effect on the stability of a hypersonic boundary layer," Computers \& Fluids, vol. 96, no. 96, pp. 350$367,2014$.

[22] Y. Zhao, W. Liu, D. Xu, D. Gang, and S. Yi, “A combined experimental and numerical investigation of roughness induced supersonic boundary layer transition," Acta Astronautica, vol. 118, pp. 199-209, 2016.

[23] P. Holloway and J. Sterett, "Effect of controlled surface roughness on boundary-layer transition and heat transfer at Mach number of 4.8 and 6.0," Tech. rep. D-2054. NASA Technical Note, Hampton, VA, USA, 1964.

[24] K. Fujii, "Experiment of the two-dimensional roughness effect on hypersonic boundary-layer transition," Journal of Spacecraft and Rockets, vol. 43, no. 4, pp. 731-738, 2006.

[25] L. Duan, X. Wang, and X. Zhong, "A high-order cut-cell method for numerical simulation of hypersonic boundarylayer instability with surface roughness," Journal of Computational Physics, vol. 229, no. 19, pp. 7207-7237, 2010.

[26] L. Duan, X. Wang, and X. Zhong, "Stabilization of a Mach 5.92 boundary layer by two-dimensional finite-height roughness," AIAA Journal, vol. 51, no. 1, pp. 266-270, 2013.

[27] C. Desjouy, S. Ollivier, O. Marsden, M. Karzova, and P. BlancBenon, "Irregular reflection of weak acoustic shock pulses on rigid boundaries: Schlieren experiments and direct numerical simulation based on a Navier-Stokes solver," Physics of Fluids, vol. 28, no. 2, article 027102, 2016.

[28] W. S. Saric, R. B. Carrillo Jr, and M. S. Reibert, "Leading-edge roughness as a transition control mechanism," in 36th AIAA Aerospace Sciences Meeting and Exhibit, Reno, NV, USA, 1998.

[29] G. S. Jiang and C. W. Shu, "Efficient implementation of weighted ENO schemes," Journal of Computational Physics, vol. 126, no. 1, pp. 202-228, 1996.

[30] C. W. ShuA. Quarteroni, "Essentially non-oscillatory and weighted essentially non-oscillatory schemes for hyperbolic conservation laws," in Advanced Numerical Approximation of Nonlinear Hyperbolic Equations, vol. 1697 of Lecture Notes in Mathematics, pp. 325-432, Springer, Berlin, Heidelberg, 1998.

[31] D. S. Balsara, S. Garain, and C. W. Shu, "An efficient class of WENO schemes with adaptive order," Journal of Computational Physics, vol. 326, pp. 780-804, 2016.

[32] J. Shi, X. Tang, Z. Wang, M. Shi, and W. Zhao, "Receptivity of boundary layer over a blunt wedge due to freestream pulse disturbances at Mach 6," International Journal of Aerospace Engineering, vol. 2016, Article ID 3196057, 14 pages, 2016.

[33] P. Balakumar, "Stability of hypersonic boundary-layers over a compression corner," in 32nd AIAA Fluid Dynamics Conference and Exhibit, St. Louis, MO, USA, June 2002.

[34] I. V. Egorov, A. V. Fedorov, and A. V. Novikov, "Numerical modeling of the disturbances of the separated flow in a rounded compression corner," Fluid Dynamics, vol. 41, no. 4, pp. 521-530, 2006.

[35] E. H. Hirschel, Basics of Aerothermodynamics, Springer, Berlin Heidelberg, 2005.

[36] K. Kara, "Receptivity of hypersonic boundary layers due to acoustic disturbances over blunt cone," in 45th AIAA Aerospace Sciences Meeting and Exhibit, Reno, NV, USA, January 2007.

[37] Y. Bandadi and A. Sbaiba, Localized Boundary Layer Receptivity To a Free Stream Acoustic Disturbance, 23ème Congrès Français de Mécanique, Lille, France, 2017.

[38] X. Liang, X. L. Li, D. X. Fu, and Y. Ma, "Effects of wall temperature on boundary layer stability over a blunt cone at Mach 7.99," Computers \& Fluids, vol. 39, no. 2, pp. 359-371, 2010.

[39] D. Fong, X. Wang, and X. Zhong, "Finite roughness effect on modal growth of a hypersonic boundary layer," in 50th AIAA Aerospace Sciences Meeting including the New Horizons Forum and Aerospace Exposition, Nashville, TN, USA, January 2012. 


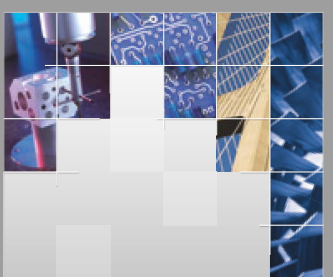

\section{Enfincering}
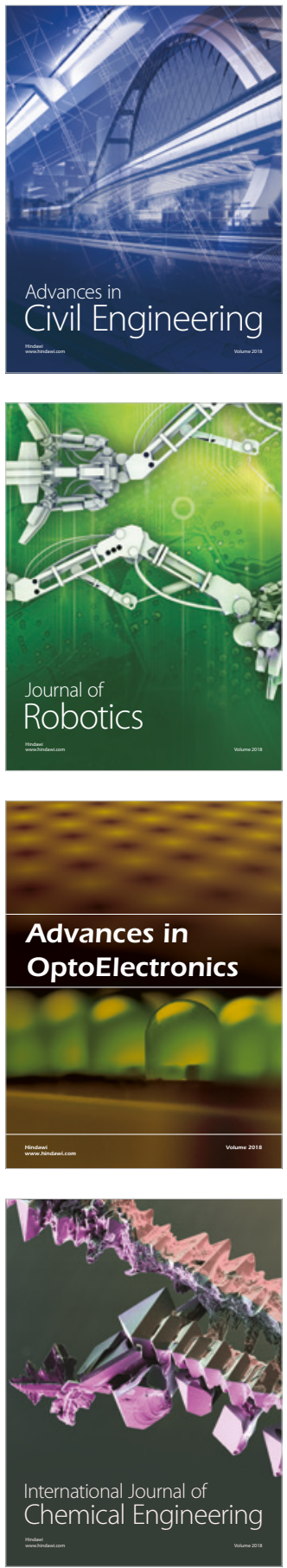

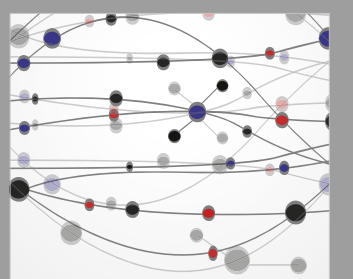

\section{Rotating \\ Machinery}

The Scientific World Journal

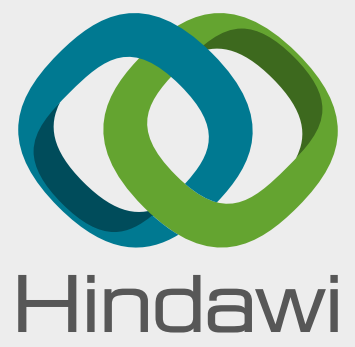

Submit your manuscripts at

www.hindawi.com
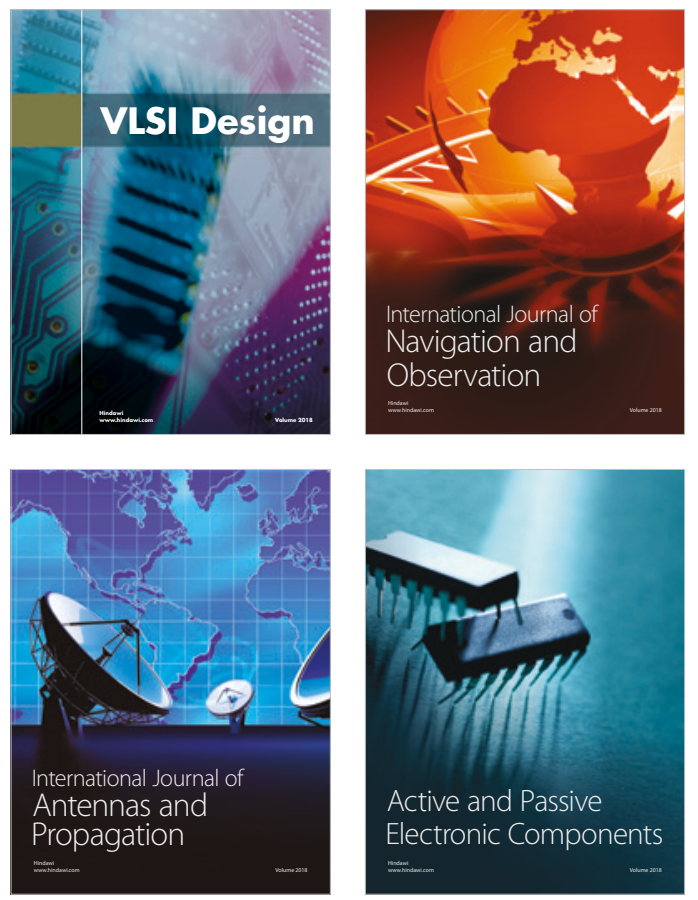
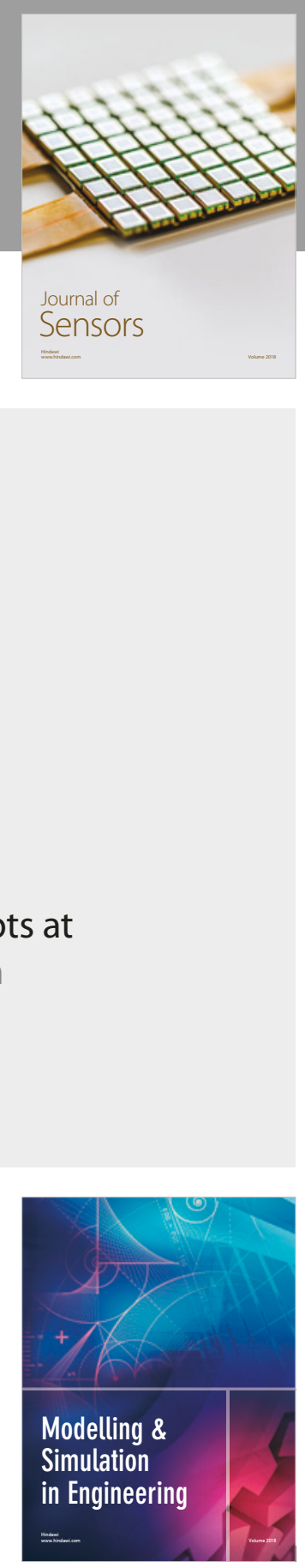

\section{Advances \\ Multimedia}
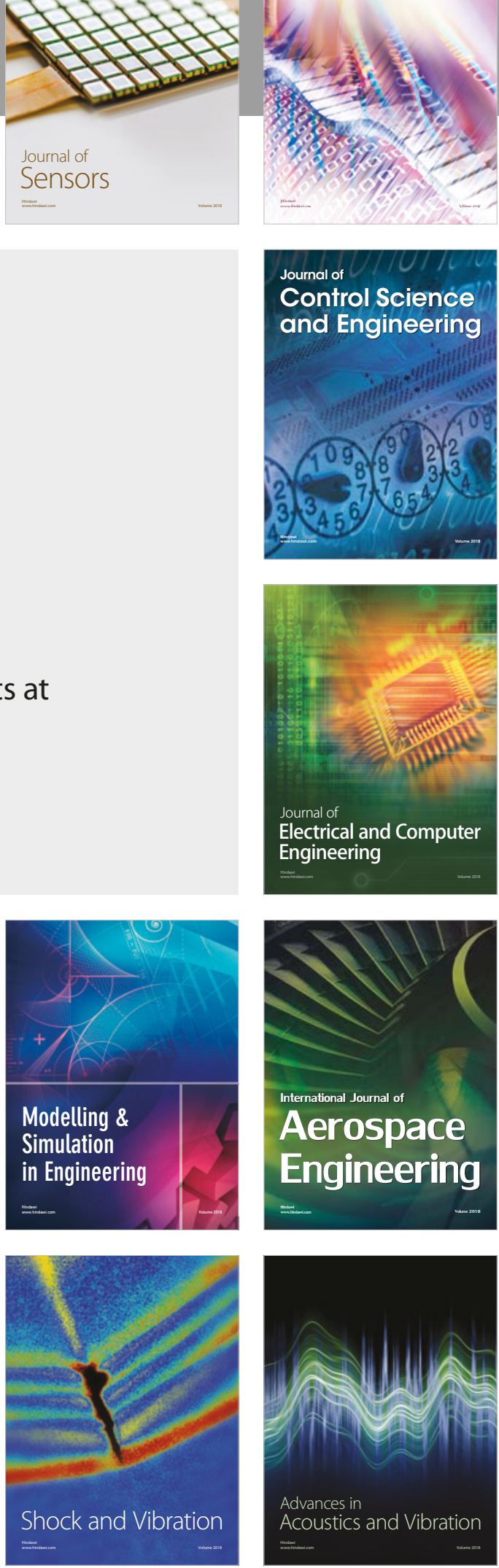